\title{
Masking-level differences in older adults: The effect of the level of the masking noise
}

\author{
MARGARET KATHLEEN PICHORA-FULLER \\ University of British Columbia, Vancouver, British Columbia, Canada \\ and \\ BRUCE A. SCHNEIDER \\ University of Toronto, Toronto, Ontario, Canada
}

\begin{abstract}
In Experiment 1, masking-level differences (MLDs) for a 500-Hz tone at five masker levels were obtained from younger and older adults. For both age groups, there were no reliable increases in MLD once the spectrum level of the masker exceeded $27 \mathrm{~dB}$ SPL. MLDs were larger for younger than for older adults over the range of masker levels tested. In Experiment 2, the levels of both the signal and the masker in one ear were attenuated by either 15 or $30 \mathrm{~dB}$ relative to their level in the other ear, which was fixed at a spectrum level of $47 \mathrm{~dB}$ SPL. MLDs for both age groups declined with increasing IAA and age-related differences were observed in all conditions. The findings of these experiments indicate that (1) age-related differences in MLDs exist even when the level of the masker is sufficiently high that older adults achieve their plateau performance, and (2) older listeners are not disadvantaged more than younger listeners by interaural differences in the level of the input.
\end{abstract}

A common complaint of older persons is difficulty in understanding speech in the noisy situations that are typical of everyday life (Working Group on Speech, 1988; Willott, 1991). A possible reason for this difficulty is that they may not be as efficient as younger adults in using interaural comparisons to "unmask" signals. For example, the threshold for detecting a monaural tone $\left(\mathrm{S}_{\mathrm{m}}\right)$ in diotic noise $\left(\mathrm{N}_{0}\right)$ is lower than the threshold for detecting the same tone in monaural noise $\left(\mathrm{N}_{\mathrm{m}}\right)$. The difference in threshold between these conditions $\left(\mathrm{S}_{\mathrm{m}} \mathrm{N}_{\mathrm{m}}-\mathrm{S}_{\mathrm{m}} \mathrm{N}_{0}\right)$ the masking-level difference (MLD) - may be smaller in older adults. In addition to these two conditions, several other conditions have also been compared to measure MLDs (for a review see Durlach \& Colburn, 1978).

In an earlier study (Pichora-Fuller \& Schneider, 1991), we looked for age-related differences in MLDs in four different dichotic listening situations. The MLDs of older listeners with good hearing were significantly (up to $5 \mathrm{~dB}$ ) smaller in all conditions. Similar differences were found by Grose, Poth, and Peters (1994) and PichoraFuller and Schneider (1992).

It is possible that the smaller MLDs of older adults were observed because the level of the masker was not suffi-

This research was supported by a grant from the Natural Sciences and Engineering Research Council of Canada. M.K.P.-F. was supported by an Ontario Ministry of Health, Health Research Personnel Development Program Fellowship (01862). We would like to thank Jane Carey for assistance in data collection, and Neil Mcmillan and three anonymous reviewers for their help with earlier versions of this manuscript. Correspondence should be addressed to M. K. Pichora-Fuller, School of Audiology and Speech Sciences, University of British Columbia, 5804 Fairview Ave., Vancouver, B.C., Canada V6T 1 Z3 (e-mail: kpf@ audiospeech.ubc.ca). ciently intense for them to have achieved maximum performance. Numerous studies have shown that the size of the MLD increases as the level of the masker increases until a plateau is reached where the size of the MLD is constant with further increases in masker level (Blodgett, Jeffress, \& Whitworth, 1962; Canahl \& Small, 1965; Diercks \& Jeffress, 1962; Dolan, 1968; Dolan \& Robinson, 1967; Hall \& Harvey, 1984, 1985; Hall, Tyler, \& Fernandes, 1983; Henning \& Zwicker, 1984; Hirsh, 1948; McFadden, 1968; Yost, 1988). For example, for the most common dichotic condition, where the masker is in phase but the noise is $180^{\circ}$ out of phase, $\mathrm{S}_{\pi} \mathrm{N}_{0}$, the MLD of normalhearing listeners for a $500-\mathrm{Hz}$ pure-tone signal in continuously presented broadband masking noise reaches a maximum of about $15 \mathrm{~dB}$ when the level of the noise exceeds a spectrum level of about 20-30 dB SPL (Hall \& Harvey, 1984). It is possible that older adults may require a higher spectrum level to achieve maximum performance. In our earlier studies (Pichora-Fuller \& Schneider. 1991, 1992), the spectrum level of the masker was held constant at $37 \mathrm{~dB}$ SPL, so we could not determine whether older listeners had acheived maximum performance. Thus, in Experiment 1 of the present study, MLDs were measured for younger and older adults as a function of masker level.

In younger listeners, MLDs are also smaller when the signal and noise presented to one ear are attenuated relative to the other ear (e.g., McFadden, 1968). To determine the relative abilities of younger and older adults to unmask signals in asymmetrical conditions, in Experiment 2, MLDs were measured for both age groups when interaural intensity differences were introduced for both signal and masker. 


\section{EXPERIMENT 1 Effect of Level of Interaurally Symmetrical Masking Noise}

\begin{abstract}
Method
Subjects. Twelve younger adults (mean age $=22.5$ years, $S D=$ 1.9 years, range $=20-26$ years) and 12 older adults (mean age $=$ 68.5 years, $S D=4.3$ years, range $=63-74$ years) participated in the present experiment. The older adults were volunteers from a pool of seniors who had participated in previous experiments. The younger subjects were university students or staff. All subjects except 1 younger subject had participated in our first study of agerelated differences in binaural unmasking (Pichora-Fuller \& Schneider, 1991). Pure-tone thresholds were obtained from each subject using standard audiometric procedures. All subjects had hearing in the better ear within normal clinical limits ( $\leq 25 \mathrm{~dB} \mathrm{HL})$ from .25 to $2 \mathrm{kHz}$, with no conductive component (air-bone gap less than $10 \mathrm{~dB}$ at each frequency), and no clinically significant asymmetry (interaural difference equal to or greater than $15 \mathrm{~dB}$ at no more than two of the test frequencies in the range from .25 to $8 \mathrm{kHz}$ ).
\end{abstract}

Stimuli and Apparatus. A $500-\mathrm{Hz}$ pure-tone signal was presented in broadband burst masking noise at each of five spectrum levels, 17, 27, 37, 47, or $52 \mathrm{~dB}$ SPL. In the baseline homophasic $\left(\mathrm{S}_{\pi} \mathrm{N}_{\pi}\right)$ conditions, the signal presented to one ear was phaseshifted by $180^{\circ}$ relative to the signal presented to the other ear, and the polarity of the masking noise presented to one ear was reversed relative to that of the masker presented to the other ear. In the comparison dichotic $\left(S_{\pi} N_{\pi \tau}\right)$ conditions, the signal was interaurally phase-shifted by $180^{\circ}$, and the masking noise presented to one ear had reversed polarity and was time-delayed by $1 \mathrm{msec}$ relative to the masking noise presented to the other ear. The $\mathrm{S}_{\pi} \mathrm{N}_{\pi}$ and $\mathrm{S}_{\pi} \mathrm{N}_{\pi \tau}$ conditions were chosen because the largest age-related differences were observed in these conditions in our earlier work (PichoraFuller \& Schneider, 1991). There were no interaural differences in the spectrum level of either the signal or the masker.

Pure-tone signals were produced by a Hewlett-Packard Model 3325 programmable function generator. The frequency and amplitude of the pure-tone signals were under software control. Bandlimited white noise $(.1-5 \mathrm{kHz})$ was produced by filtering (Wavetek System 716 Brickwall Filter, rolloff $=115 \mathrm{~dB} /$ octave) the output of a noise generator (General Radio 1381). To allow for the possibility of time-delaying the noise, it was then digitized at a rate of $40 \mathrm{kHz}$ and stored in a buffer that was continually updated. This digital noise was sent to two 16-bit digital-to-analog converters. The noise sent to one of the digital-to-analog converters could be delayed by multiples of $.025 \mathrm{msec}$ relative to the noise sent to the other converter by accessing different points in the buffer. When the noise was time-delayed, the delay, $\tau$, in the masking noise was equal to $1 \mathrm{msec}$, half of the period of the pure-tone signal $[1 /(2 \tau)=$ $500 \mathrm{~Hz}] .{ }^{1}$ The noise output from one of the converters was added to the pure tone going to the left ear, and the noise output from the other converter was added to the pure tone going to the right ear. By reversing the polarity of one earphone relative to the other earphone, the signal and masker presented to one ear were phase-reversed relative to the signal and masker presented to the other ear. The masking noise and the tone, when present, were gated on and off simultaneously (200-msec duration with 10 -msec rise-decay time). Stimuli were presented over matched TDH-49 earphones in a singlewall sound-attenuating booth. The presentation and timing of events were managed by a computer.

Procedure. A two-interval, two-alternative, forced-choice (2I2$\mathrm{AFC}$ ) procedure was employed. Each trial consisted of two observation intervals, one of which contained the signal. Four signal levels spaced $6 \mathrm{~dB}$ apart were employed. The range of the psychometric function for an experienced subject in these conditions is around
10-12 dB (Green, 1966). Because our subjects were relatively inexperienced, we decided to employ a range that was $50 \%$ larger. Furthermore, by mixing signal levels within a condition, the cooperation of the subjects was readily obtained, whereas if the signal levels had been blocked, some subjects would have been reluctant to complete blocks in which all the signals were below threshold. The actual signal levels employed depended on the subject and on the condition and were selected so that values on the psychometric function between chance and perfect performance were obtained.

The signal interval was randomly selected on each trial. Subjects initiated a trial by pressing a button and then, $500 \mathrm{msec}$ later, the first of the two observation intervals was presented, followed by a 500 -msec separation between intervals. Subjects indicated which interval they thought contained the signal by pressing one of two buttons corresponding to the two intervals. Signal lights inside the booth marked the beginning of the trial and each interval and also provided immediate feedback to the subjects as to whether each response had been correct or incorrect.

Each of the 10 conditions ( 2 types of presentation $\left[\mathrm{S}_{\pi} \mathrm{N}_{\pi}\right.$ and $\left.\mathrm{S}_{\pi} \mathrm{N}_{\pi \tau}\right] \times$ five masker spectrum levels) consisted of 320 trials. Thus, in each condition, each of the four signal levels was presented 80 times. Each condition took about $25 \mathrm{~min}$ to complete. Subjects were tested individually, and at each session they usually completed two conditions with a break between conditions. In the event that the signal leveis tested did not yield values covering the range of the psychometric function between chance and perfect performance, the condition was repeated with a different set of four signal levels that were then used in the analysis. All subjects completed all conditions. Prior to the 320 test trials in each condition, each subject experienced 32 practice trials with two signal intensities well above threshold (in all cases, the percentage of correct responses exceeded $90 \%$ on practice trials).

\section{Results}

Logistic functions, fit to the data points using the nonlinear least squares routine in Mathematica (Wolfram, 1991), were used to describe how percent correct detection changed as a function of stimulus intensity for each subject in each condition. Threshold was defined as the stimulus level corresponding to $75 \%$ correct. Figure 1 (top panel) plots average detection thresholds for the younger and older adults in the $\mathrm{S}_{\pi} \mathrm{N}_{\pi}$ and $\mathrm{S}_{\pi} \mathrm{N}_{\pi \tau}$ conditions as a function of the spectrum level of the noise masker. The corresponding data for individual subjects are presented in Table 1.

Figure 1 shows that the average $\mathrm{S}_{\pi} \mathrm{N}_{\pi}$ and $\mathrm{S}_{\pi} \mathrm{N}_{\pi \tau}$ thresholds for the younger adults were better than those for the older adults in all conditions. The thresholds obtained in the $\mathrm{S}_{\pi} \mathrm{N}_{\pi \tau}$ conditions were subtracted from the corresponding thresholds obtained in the $\mathrm{S}_{\pi} \mathrm{N}_{\pi}$ conditions to obtain the MLDs shown in the lower panel of Figure 1. The average MLDs for the younger group exceeded the average MLDs for the older group. An analysis of variance (ANOVA) for MLD confirmed this description with significant effects of both age $[F(1,22)=$ $24.89, p<.001]$ and masker level $[F(4,88)=10.46, p<$ $.001]$, but with no significant age $\times$ masker level interaction $[F(4,88)=1.47, p>.05]$. A Student-NewmanKeuls test confirmed that the mean MLD obtained when the masking noise was presented at a spectrum level of $17 \mathrm{~dB}$ SPL was significantly smaller $(p<.01)$ than the 


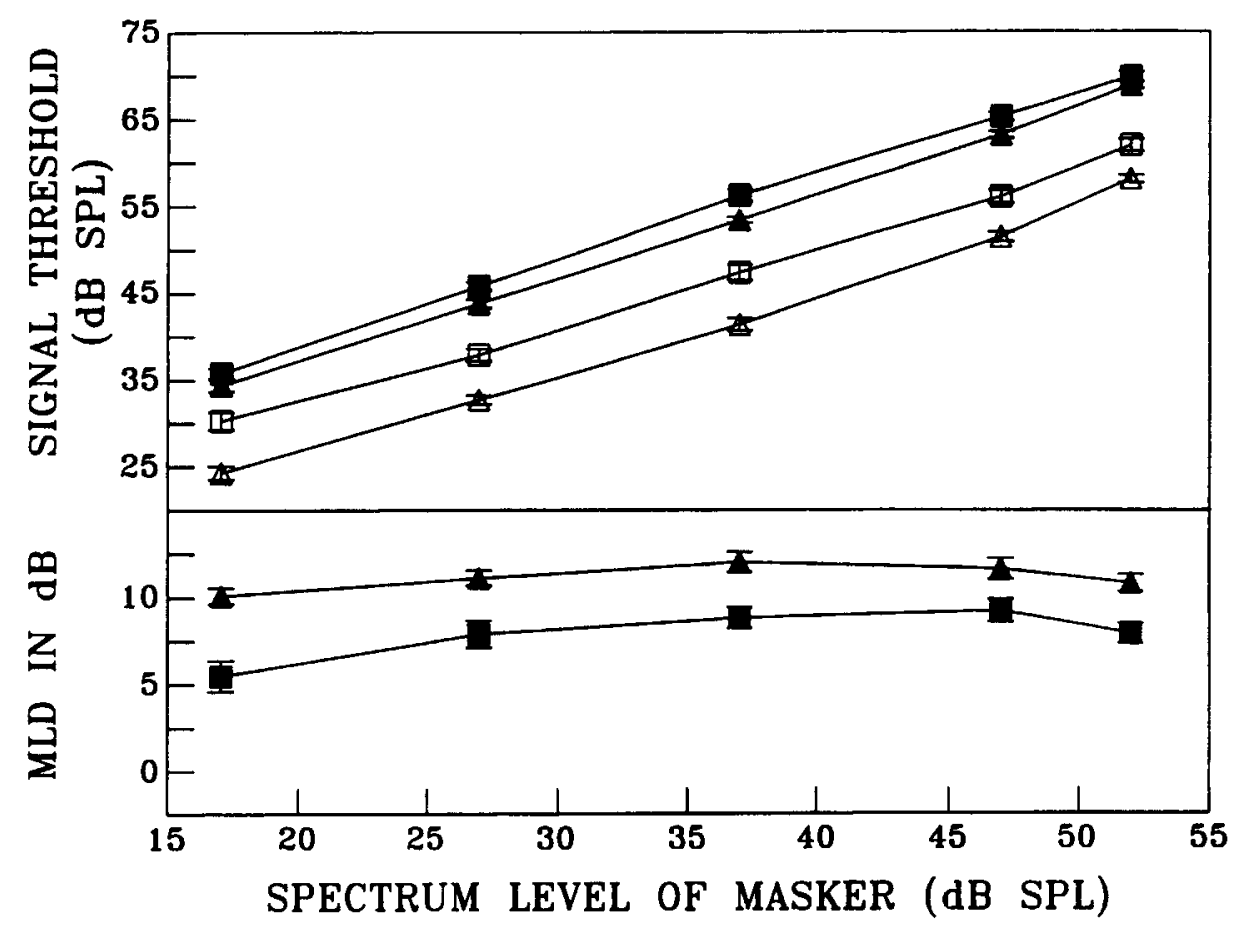

Figure 1. Top: Average $S_{\pi} N_{\pi}$ and $S_{\pi} N_{\pi}$ thresholds for the younger group (filled and unfilled triangles, respectively) and for the older group (filled and unfilled squares, respectively) as a function of the spectrum level of the masking noise. Vertical bars show $\pm 1 S E$. Bottom: Average masking-level differences (MLDs) as a function of the spectrum level of the masker for younger (triangles) and older (squares) listeners. Vertical bars show $\pm 1 S E$.

mean MLDs obtained when the masking noise was presented at the four higher spectrum levels, which did not differ significantly from each other.

The finding that the mean size of the MLD was significantly smaller when the masking noise was presented at the lowest spectrum level raises the question of whether or not there was a floor effect for some listeners. As the spectrum level of the masker decreases, both the $\mathrm{S}_{\pi} \mathrm{N}_{\pi \tau}$ and $\mathrm{S}_{\pi} \mathrm{N}_{\pi}$ thresholds are, in effect, limited by the $\mathrm{S}_{\pi}$ threshold (the threshold in quiet for a pure tone presented with an interaural phase difference of $180^{\circ}$ ) since both $\mathrm{S}_{\pi} \mathrm{N}_{\pi \tau}$ and $\mathrm{S}_{\pi} \mathrm{N}_{\pi}$ become $\mathrm{S}_{\pi}$ as noise power goes to zero. The same $2 \mathrm{I} 2 \mathrm{AFC}$ procedure that had been used to measure masked binaural thresholds was used to determine $S_{\pi}$ threshold for each listener (see Table 2). A comparison of Tables 1 and 2 indicates that for 2 of the older adults (A.B. and J.Y.), the $\mathrm{S}_{\pi} \mathrm{N}_{\pi \tau}$ thresholds in the $27 \mathrm{~dB}$ SPL masker condition were less than $10 \mathrm{~dB}$ above their corresponding $S_{\pi}$ thresholds. For these two listeners, then, it is unlikely that a $10-\mathrm{dB}$ decrease in the masker level from 27 to $17 \mathrm{~dB}$ SPL could produce a $10-\mathrm{dB}$ decrease in $\mathrm{S}_{\pi} \mathrm{N}_{\pi \tau}$ thresholds. Such a limitation could lead to a correlation between $\mathrm{S}_{\pi} \mathrm{N}_{\pi \tau}$ and $\mathrm{S}_{\pi}$ thresholds at the lower masker levels but not necessarily at the higher masker levels. Correlation coefficients between $S_{\pi} N_{\pi}$ and $S_{\pi}$ were determined for each level of the masker for younger and older adults. A significant correlation $(\alpha=$
.05 , Bonferonni corrected, see Table 3 ) was observed for the older adults at a masker spectrum level of $17 \mathrm{~dB}$ SPL. None of the other correlation coefficients were significant. These results suggest that the size of the MLDs of older adults at the lowest spectrum level may be limited by their $\mathrm{S}_{\pi}$ thresholds.

We also investigated whether the MLDs of either younger or older adults were related to their audiometric status. To obtain threshold estimates at $500 \mathrm{~Hz}$ that were more accurate than those obtained using clinical audiometry, the $500-\mathrm{Hz}$ thresholds in quiet were remeasured using the 2I2AFC procedure (Table 2). Left- and rightear thresholds were then averaged to obtain an index of audiometric status at $500 \mathrm{~Hz}$. Correlations between audiometric status at $500 \mathrm{~Hz}$ and MLDs are presented in Table 3. Clearly, there is no indication of a correlation between audiometric status and the size of MLD in any of the masking conditions for the younger adults. The correlation coefficients for the older adults were all negative and they were fairly high in the 17,27 , and $47 \mathrm{~dB}$ SPL masker conditions, but they did not reach significance once the Bonferonni correction for multiple tests was applied.

To determine whether MLDs were correlated with highfrequency hearing loss, audiometric thresholds at 3,4, and $8 \mathrm{kHz}$ in the left and right ears were averaged to obtain an index of high-frequency hearing loss for each listener. Correlations between these pure-tone averages and 
Table 1

Individual $S_{\pi} \mathbf{N}_{\pi}$ and $S_{\pi} N_{\pi \tau}$ Thresholds (dB SPL) $\times$ Masking Level Condition for Subjects in Experiment I

\begin{tabular}{|c|c|c|c|c|c|c|c|c|c|c|c|}
\hline \multirow[b]{3}{*}{ Group } & \multirow[b]{3}{*}{ Subject } & \multicolumn{10}{|c|}{ Condition } \\
\hline & & \multicolumn{2}{|c|}{$17 \mathrm{~dB} \mathrm{SPL}$} & \multicolumn{2}{|c|}{$27 \mathrm{~dB}$ SPL } & \multicolumn{2}{|c|}{$37 \mathrm{~dB}$ SPL } & \multicolumn{2}{|c|}{$47 \mathrm{~dB}$ SPL } & \multicolumn{2}{|c|}{$52 \mathrm{~dB} S P L$} \\
\hline & & $\mathrm{S}_{\pi} \mathrm{N}_{\pi}$ & $\mathrm{S}_{\pi} \mathrm{N}_{\pi \tau}$ & $\mathrm{S}_{\pi} \mathrm{N}_{\pi}$ & $\mathrm{S}_{\pi} \mathrm{N}_{\pi \tau}$ & $\mathrm{S}_{\pi} \mathrm{N}_{\pi}$ & $\mathrm{S}_{\pi} \mathrm{N}_{\pi \tau}$ & $\mathrm{S}_{\pi} \mathrm{N}_{\pi}$ & $\mathrm{S}_{\pi} \mathrm{N}_{\pi \tau}$ & $\mathrm{S}_{\pi} \mathrm{N}_{\pi}$ & $\mathrm{S}_{\pi} \mathrm{N}_{\pi \tau}$ \\
\hline \multirow[t]{13}{*}{ Older } & P.F. & 34.8 & 27.9 & 43.7 & 36.2 & 54.5 & 45.2 & 64.4 & 54.3 & 68.4 & 61.1 \\
\hline & H.H. & 34.6 & 33.9 & 46.4 & 37.7 & 58.1 & 49.6 & 65.7 & 58.6 & 72.9 & 63.3 \\
\hline & A.B. & 40.1 & 34.9 & 44.7 & 38.1 & 53.0 & 45.9 & 62.9 & 54.5 & 68.2 & 58.9 \\
\hline & P.P. & 35.9 & 29.6 & 44.6 & 40.7 & 60.2 & 55.1 & 66.0 & 58.5 & 70.2 & 65.3 \\
\hline & C.D. & 35.3 & 30.8 & 46.0 & 37.8 & 57.5 & 47.2 & 66.1 & 55.2 & 71.9 & 63.1 \\
\hline & V.W. & 35.8 & 26.0 & 45.5 & 34.2 & 57.1 & 45.0 & 65.5 & 53.8 & 68.2 & 58.9 \\
\hline & R.L. & 37.6 & 31.5 & 48.9 & 35.6 & 58.8 & 49.7 & 66.1 & 55.7 & 69.7 & 61.2 \\
\hline & C.M. & 37.3 & 32.9 & 48.2 & 42.9 & 56.9 & 50.1 & 67.4 & 62.2 & 71.1 & 65.4 \\
\hline & L.D. & 34.5 & 23.3 & 46.0 & 36.4 & 56.3 & 45.1 & 66.9 & 55.0 & 72.6 & 63.0 \\
\hline & J.L. & 35.9 & 29.2 & 44.7 & 39.8 & 54.4 & 45.1 & 65.0 & 53.1 & 69.4 & 60.0 \\
\hline & D.F. & 32.1 & 29.4 & 44.2 & 36.5 & 51.8 & 45.4 & 62.0 & 54.3 & 67.4 & 58.6 \\
\hline & J.Y. & 35.9 & 34.5 & 46.2 & 38.7 & 56.2 & 45.6 & 64.3 & 56.9 & 68.1 & 64.0 \\
\hline & Mean & 35.8 & 30.3 & 45.8 & 37.9 & 56.2 & 47.4 & 65.2 & 56.0 & 69.8 & 61.9 \\
\hline \multirow[t]{13}{*}{ Younger } & R.R. & 33.2 & 20.3 & 41.8 & 29.0 & 52.1 & 38.6 & 61.8 & 48.7 & 68.3 & 55.8 \\
\hline & J.F. & 31.5 & 24.9 & 43.1 & 34.9 & 54.6 & 46.2 & 62.9 & 54.6 & 69.5 & 59.4 \\
\hline & M.M. & 35.0 & 24.4 & 46.3 & 34.6 & 54.3 & 43.4 & 64.3 & 52.2 & 69.2 & 58.8 \\
\hline & M.S. & 32.5 & 20.5 & 41.1 & 31.4 & 53.3 & 41.9 & 60.9 & 51.3 & 67.8 & 55.8 \\
\hline & S.K. & 37.7 & 27.8 & 42.4 & 33.3 & 53.5 & 42.4 & 60.9 & 51.6 & 70.6 & 59.2 \\
\hline & N.M. & 33.4 & 23.6 & 44.0 & 32.3 & 54.0 & 44.8 & 63.6 & 55.3 & 66.6 & 58.9 \\
\hline & A.L. & 35.0 & 27.1 & 45.2 & 34.5 & 51.2 & 39.5 & 63.5 & 50.3 & 69.0 & 55.5 \\
\hline & B.L. & 40.3 & 29.0 & 45.9 & 34.8 & 53.8 & 41.5 & 64.8 & 51.5 & 70.6 & 59.1 \\
\hline & G.H. & 32.5 & 22.8 & 42.3 & 31.8 & 52.2 & 38.8 & 63.8 & 49.5 & 66.4 & 58.0 \\
\hline & G.K. & 33.9 & 23.9 & 44.7 & 32.3 & 55.1 & 40.5 & 64.2 & 51.2 & 70.2 & 60.0 \\
\hline & C.S. & 35.3 & 25.2 & 45.2 & 32.2 & 53.2 & 38.5 & 63.1 & 50.3 & 69.8 & 58.1 \\
\hline & D.C. & 32.5 & 22.5 & 43.6 & 31.0 & 52.9 & 40.3 & 62.9 & 50.7 & 68.0 & 58.4 \\
\hline & Mean & 34.4 & 24.3 & 43.8 & 32.7 & 53.4 & 41.4 & 63.1 & 51.4 & 68.8 & 58.1 \\
\hline
\end{tabular}

MLDs are also presented in Table 3 for both younger and older adults. For the younger adults, the correlations between the high-frequency pure-tone average and the MLD were small and more often positive than negative. For older adults, there was a significant correlation when the spectrum level of the noise was $27 \mathrm{~dB}$ SPL; correlations at the other frequencies, although they were moderately high and negative, were not significant.

\section{Discussion}

For all spectrum levels of the broadband burst masker there was a significant (between 2.8 and $4.6 \mathrm{~dB}$ ) age effect on MLD. For both age groups, the average size of the MLDs obtained when the masking noise was presented at a spectrum level of $17 \mathrm{~dB}$ SPL was smaller than the size of the MLDs obtained at higher masker levels; however, MLDs reached a plateau value when the masking noise was presented at a spectrum level of at least $27 \mathrm{~dB}$ SPL. Thus, consistent with previous studies of the effect of masker level on the size of the MLD, both younger and older adults reached a plateau beyond which further increases in masking level produced no further increases in MLD. Clearly, age-related differences in MLDs were observed even in masking conditions where there is no doubt that older adults achieved their plateau level of performance.

At very low levels of the masker, the performance of subjects in dichotic conditions is ultimately limited by their $S_{\pi}$ thresholds in the sense that their $S_{\pi} N_{\pi \tau}$ thresh- olds cannot be less than their $\mathrm{S}_{\pi}$ thresholds when there is no external noise. Therefore, for sufficiently low levels of the masker (levels near the absolute threshold for detection of the masker), we might expect to find correlations between $\mathrm{S}_{\pi}$ and $\mathrm{S}_{\pi} \mathrm{N}_{\pi \tau}$ thresholds. A statistically significant correlation was found for older (but not younger) subjects at a masker spectrum level of $17 \mathrm{~dB}$ SPL, supporting the hypothesis that the performance of older adults at the lowest masker level was limited by $\mathrm{S}_{\pi}$ threshold.

A consistent pattern of negative correlations between MLDs and high-frequency thresholds for older adults would suggest that the same factors responsible for their high-frequency hearing loss might also be responsible for reduced MLDs at $500 \mathrm{~Hz}$. A significant Bonferonniadjusted correlation was found between their average highfrequency pure-tone average thresholds and MLDs at a masker spectrum level of $27 \mathrm{~dB}$ SPL, and moderate although not significant correlations were found at other spectrum levels. Thus the overall pattern of correlations provides weak evidence for the possibility that the same factors contributing to high-frequency hearing loss may also be responsible for reduced MLDs at $500 \mathrm{~Hz}$. It is also possible that high-frequency hearing loss may simply be an indicator of a general deterioration in auditory processing and the specific anatomical and/or physiological factors responsible for high-frequency hearing loss could differ from those responsible for reduced MLDs at low frequencies. 
Table 2

$S_{\pi}$, and Right- and Left-Ear Monaural Thresholds (dB SPL), Obtained at 500-Hz for Each Subject in Quiet Using a 212AFC Procedure

\begin{tabular}{|c|c|c|c|}
\hline Subject & $\mathrm{S}_{\pi}$ & Right & Left \\
\hline \multicolumn{4}{|c|}{ Older Group } \\
\hline P.F. & 17.0 & 20.7 & 25.9 \\
\hline H.H. & 21.4 & 28.4 & 29.0 \\
\hline A.B. & 35.0 & 38.6 & 44.7 \\
\hline P.P. & 20.2 & 25.3 & 19.0 \\
\hline C.D. & 24.0 & 30.3 & 26.4 \\
\hline V.W. & 12.5 & 14.4 & 13.6 \\
\hline R.L. & 9.2 & 12.5 & 13.5 \\
\hline C.M. & 29.6 & 26.7 & 44.8 \\
\hline L.D. & 13.2 & 14.5 & 18.2 \\
\hline J.L. & 21.3 & 23.8 & 25.5 \\
\hline D.F. & 22.6 & 26.8 & 26.4 \\
\hline J.Y. & 29.7 & 33.5 & 26.7 \\
\hline Mean & 21.3 & 24.6 & 26.1 \\
\hline \multicolumn{4}{|c|}{ Younger Group } \\
\hline R.R. & 4.0 & 6.3 & 5.0 \\
\hline J.F. & 7.2 & 7.0 & 10.4 \\
\hline M.M. & 14.3 & 20.4 & 15.3 \\
\hline M.S. & 17.5 & 18.0 & 15.8 \\
\hline S.K. & 7.6 & 13.6 & 9.5 \\
\hline N.M. & 12.3 & 8.1 & 14.6 \\
\hline A.L. & 22.0 & 21.1 & 28.7 \\
\hline B.L. & 8.7 & 17.6 & 11.0 \\
\hline G.H. & 18.4 & 21.8 & 20.1 \\
\hline G.K. & 19.4 & 17.6 & 17.3 \\
\hline C.S. & 6.5 & 9.1 & 8.1 \\
\hline D.C. & 5.6 & 14.0 & 9.3 \\
\hline Mean & 11.9 & 14.5 & 13.8 \\
\hline
\end{tabular}

\section{EXPERIMENT 2 \\ Effect of Interaural Level Differences on the MLD}

Asymmetries in the level of presentation of masker and signal result in a reduction in the size of the MLD in normal-hearing younger adults (McFadden, 1968). In Experiment 2 , we compared the effect of interaural attenuation (IAA) on the MLDs of younger and older adults.

\section{Method}

The same subjects served in Experiment 2 as in Experiment 1. The same equipment and procedures were used. The spectrum level of the masking noise was fixed at $47 \mathrm{~dB}$ SPL in the right ear. However, the sound pressure level of both masker and signal in the left ear were reduced by either 15 or $30 \mathrm{~dB}$ to produce an IAA difference. The two conditions in which there was IAA in both the signal and the masker are analogous, respectively, to the subject having a $15-$ or $30-\mathrm{dB}$ asymmetrical conductive hearing loss, with the left ear being less sensitive than the right ear. There were a total of six conditions (two types of masker presentation $\left[\mathrm{S}_{\pi} \mathrm{N}_{\pi}\right.$ or $\left.\mathrm{S}_{\pi} \mathrm{N}_{\pi \tau}\right] \times$ three levels of IAA $[0,15$, and $30 \mathrm{~dB}])^{2}{ }^{2}$ The data for the $0-\mathrm{dB}$ interaural condition (symmetrical masking at a spectrum level of $47 \mathrm{~dB}$ SPL) were those obtained in Experiment 1.

\section{Results}

In Figure 2 (top panel), the average thresholds of the signals presented to the unattenuated ear are plotted as a function of IAA for both groups in the $\mathrm{S}_{\pi} \mathrm{N}_{\pi}$ and $\mathrm{S}_{\pi} \mathrm{N}_{\pi \tau}$ conditions. Individual thresholds are presented in Table 4.

In all IAA conditions, $\mathrm{S}_{\pi} \mathrm{N}_{\pi}$ and $\mathrm{S}_{\pi} \mathrm{N}_{\pi \tau}$ thresholds were lower for the younger adults than for the older adults. For both groups, the diotic thresholds remained constant, whereas dichotic thresholds increased as IAA increased. As shown in the bottom panel of Figure 2, for both age groups, MLDs decreased with increased IAA. The MLDs of the older subjects appear to be smaller than the MLDs of the younger subjects in the 0- and 30$\mathrm{dB}$ IAA conditions and nearly equivalent in the $15-\mathrm{dB}$ IAA condition. However, an ANOVA failed to reveal a significant age $\times$ IAA condition interaction $[F(2,44)=$ $2.15, p>.1]$. There was, however, a significant main effect of IAA $[F(2,44)=65.9, p<.0001]$ and a significant main effect of age $[F(1,22)=5.95, p<.05]$.

It is possible that some subjects may have had $500-\mathrm{Hz}$ threshold asymmetries that compounded or offset the experimentally introduced attenuation. To investigate this possibility, for each age group, correlations were computed between the size of the right-left ear difference in $500-\mathrm{Hz}$ quiet threshold and the size of the MLD in the three IAA conditions. Of the six correlations that were measured $(0.28,-0.07$, and 0.42 for younger adults and $0.26,-0.07$, and 0.30 for older adults in the $0-, 15-$, and 30-dB IAA conditions, respectively), none were significant. Therefore, it seems that degree of threshold asymmetry did not account for the individual differences in MLDs in these subjects.

\section{Discussion}

An interaural level difference of 15 or $30 \mathrm{~dB}$ in both the signal and the masker, which preserved the same external signal-to-noise ratio in both the unattenuated presentation to one ear and the attenuated presentation to the other ear, had no effect on the mean $\mathrm{S}_{\pi} \mathrm{N}_{\pi}$ threshold of either age group. In contrast, $\mathrm{S}_{\pi} \mathrm{N}_{\pi \tau}$ threshold increased significantly as IAA increased from 0 to $15 \mathrm{~dB}$ and from 15 to $30 \mathrm{~dB}$. For the younger subjects, the extent of the increase in $\mathrm{S}_{\pi} \mathrm{N}_{\pi \tau}$ threshold with increasing IAA was virtually identical to that observed by Colburn and Latimer (1978), for the condition in their experiment that most closely matches our $\mathrm{S}_{\pi} \mathrm{N}_{\pi \tau}$ condition. ${ }^{3}$ The decibel increases in dichotic threshold produced by attenuating the input to one ear for the younger and older adults in this experiment, and for the equivalent condition for subjects in the experiment of Colburn and Latimer, are shown in Figure 3. The decibel increase for the older adults appears to be slightly smaller than that for the younger adults but follows the same basic pattern.

\section{GENERAL DISCUSSION AND CONCLUSIONS}

In Experiment 1, the MLDs of older adults were consistently smaller than those of younger adults for noise spec- 
Table 3

Correlations Between $S_{\pi}$ and $S_{\pi} N_{\pi \tau}$ Thresholds, and Between the Average of the Left- and Right-Ear 500-Hz and High-Frequency Average Thresholds and MLDs $\times$ Masking Level for Younger and Older Adults

\begin{tabular}{|c|c|c|c|c|c|c|}
\hline \multirow{2}{*}{$\begin{array}{l}\text { Spectrum Level of } \\
\text { Masker (dB SPL) }\end{array}$} & \multicolumn{2}{|c|}{$\mathrm{S}_{\pi}$ and $\mathrm{S}_{\pi} \mathrm{N}_{\pi \tau}$} & \multicolumn{2}{|c|}{ 500-Hz Threshold and MLD } & \multicolumn{2}{|c|}{$\begin{array}{l}\text { High-Frequency Pure-Tone } \\
\text { Average and MLD }\end{array}$} \\
\hline & Younger & Older & Younger & Older & Younger & Older \\
\hline 17 & 0.00 & $0.71^{*}$ & -0.22 & -0.60 & -0.37 & -0.40 \\
\hline 27 & 0.24 & 0.62 & -0.16 & -0.61 & 0.01 & $-0.85^{*}$ \\
\hline 37 & -0.10 & -0.01 & 0.08 & -0.46 & -0.06 & -0.55 \\
\hline 47 & -0.05 & 0.32 & 0.37 & -0.63 & 0.14 & -0.42 \\
\hline 52 & -0.20 & 0.15 & 0.07 & -0.20 & 0.15 & -0.43 \\
\hline
\end{tabular}

*For each group in each masking-level condition, $\mathrm{S}_{\pi}$ thresholds were correlated with $\mathrm{S}_{\pi} \mathrm{N}_{\pi}$ thresholds, and $500-\mathrm{Hz}$ thresholds (left- and right-ear average) and the high-frequency pure-tone averages (average of 3-, 4-, and 8-kHz thresholds in the left and right ears) were correlated with masking-level difference (MLD). Applying the Bonferonni correction for five independent tests, $|\mathbf{r}|$ must be at least .71 to reach significance $(\alpha=.05, d f=10$, two-tailed).

trum levels ranging from 17 to $52 \mathrm{~dB}$ SPL, ruling out the possibility that the MLDs of older listeners were smaller than those of younger listeners because the masker was presented at a level that was insufficient for the older adults to achieve their plateau level of performance. No significant correlations (using the Bonferonni correction) were observed between MLDs and the average monaural thresholds at $500 \mathrm{~Hz}$ for any of the five levels of the masker for either group. However, a significant correlation between MLD and average high-frequency audiometric threshold was observed for the older group when the masker spectrum level was $27 \mathrm{~dB}$ SPL. Thus, there is some indica- tion of an association between the size of the MLD and the degree of high-frequency hearing loss for older adults in the earliest stages of presbycusis.

When the input to the two ears was asymmetrical, agerelated differences were also observed. These age-related differences could not be attributed to threshold asymmetries in quiet because there were no significant correlations between threshold asymmetries and MLDs. These results suggest that binaural unmasking by older adults would not necessarily be any more compromised by audiometric asymmetries than would be the case for younger adults.

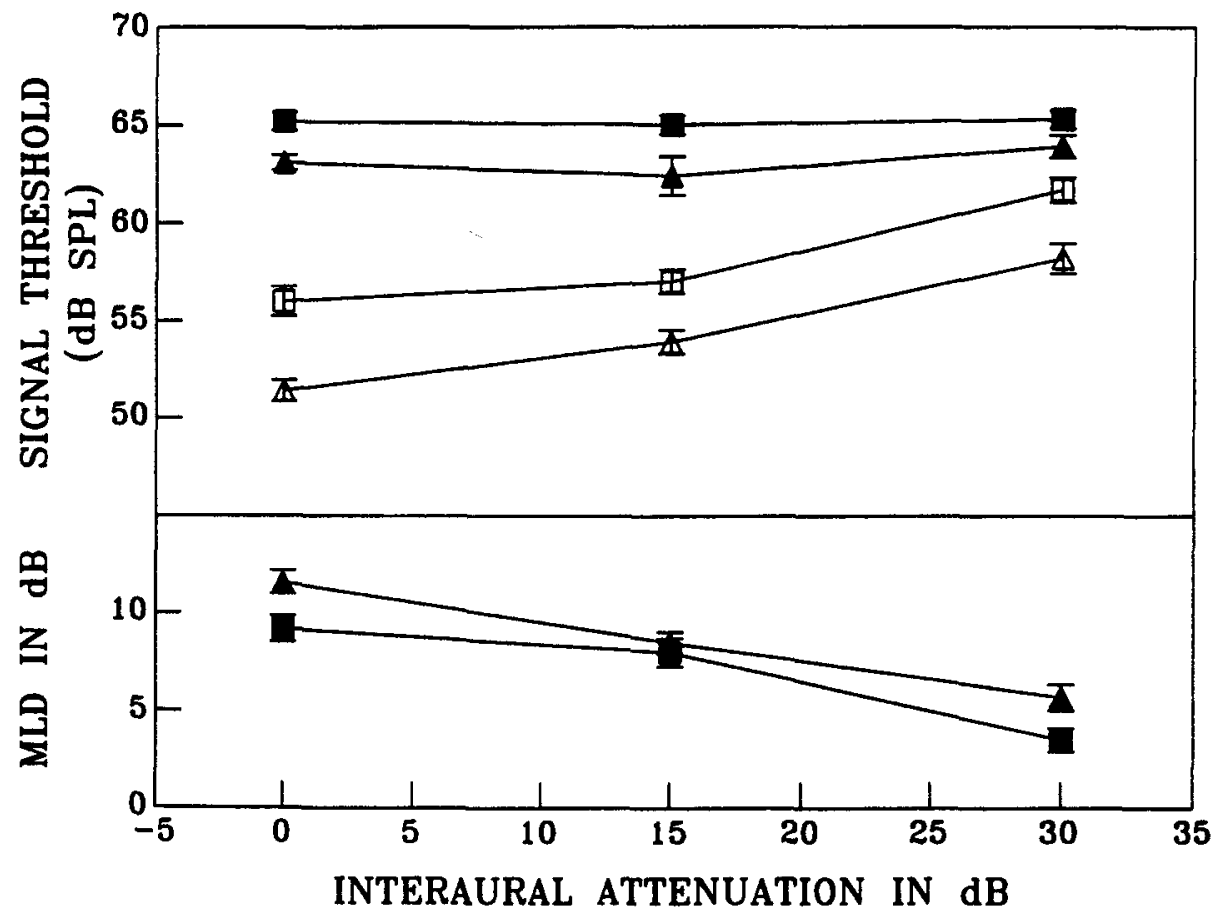

Figure 2. Top: Average $S_{\pi} N_{\pi}$ and $S_{\pi} N_{\pi \tau}$ thresholds (sound pressure level for $75 \%$ correct detection) for the younger group (filled and unfilled triangles, respectively) and for the older group (filled and unfilled squares, respectively) as a function of the amount of interaural attenuation. Vertical bars show $\pm 1 S E$. Bottom: Average masking-level differences (MLDs) as a function of interaural attenuation for younger (triangles) and older (squares) listeners. Vertical bars show $\pm 1 S E$. 
Table 4

Individual Thresholds (Unattenuated Ear) in dB SPL by Attenuation Condition for the Subjects in Experiment 2

\begin{tabular}{|c|c|c|c|c|c|c|}
\hline \multirow[b]{2}{*}{ Subject } & \multicolumn{2}{|c|}{$0-\mathrm{dB}$ IAA } & \multicolumn{2}{|c|}{ 15-dB IAA } & \multicolumn{2}{|c|}{ 30-dB IAA } \\
\hline & $\mathrm{S}_{\pi} \mathrm{N}_{\pi}$ & $\mathrm{S}_{\pi} \mathrm{N}_{\pi \tau}$ & $\mathrm{S}_{\pi} \mathrm{N}_{\pi}$ & $\mathrm{S}_{\pi} \mathrm{N}_{\pi t}$ & $\mathrm{~S}_{\pi} \mathrm{N}_{\pi}$ & $\mathrm{S}_{\pi} \mathrm{N}_{\pi}$ \\
\hline \multicolumn{7}{|c|}{ Older Group } \\
\hline P.F. & 64.4 & 54.3 & 65.0 & 55.8 & 64.5 & 59.9 \\
\hline H.H. & 65.7 & 58.6 & 67.1 & 57.8 & 67.6 & 61.9 \\
\hline A.B. & 62.9 & 54.5 & 66.2 & 61.1 & 64.7 & 65.3 \\
\hline P.P. & 66.0 & 58.5 & 62.2 & 57.8 & 64.5 & 61.4 \\
\hline C.D. & 66.1 & 55.2 & 66.4 & 56.8 & 67.0 & 61.8 \\
\hline V.W. & 65.5 & 53.8 & 64.9 & 54.3 & 62.4 & 58.1 \\
\hline R.L. & 66.2 & 55.7 & 65.5 & 57.3 & 67.2 & 63.6 \\
\hline C.M. & 67.4 & 62.2 & 67.1 & 59.9 & 64.9 & 63.3 \\
\hline L.D. & 67.0 & 55.0 & 65.7 & 53.7 & 66.0 & 58.6 \\
\hline J.L. & 65.2 & 53.1 & 64.6 & 55.3 & 66.4 & 64.4 \\
\hline D.F. & 62.0 & 54.3 & 61.9 & 58.0 & 62.8 & 60.8 \\
\hline J.Y. & 64.3 & 56.9 & 63.8 & 56.5 & 65.2 & 61.4 \\
\hline Mean & 65.2 & 56.0 & 65.0 & 57.0 & 65.3 & 61.7 \\
\hline \multicolumn{7}{|c|}{ Younger Group } \\
\hline R.R. & 61.8 & 48.7 & 61.5 & 51.4 & 62.5 & 56.4 \\
\hline J.F. & 62.9 & 54.7 & 64.0 & 54.2 & 63.9 & 57.9 \\
\hline M.M. & 64.3 & 52.2 & 64.2 & 53.3 & 66.4 & 59.5 \\
\hline M.S. & 60.9 & 51.3 & 59.6 & 52.2 & 61.5 & 54.7 \\
\hline S.K. & 60.9 & 51.7 & 65.6 & 56.5 & 65.2 & 58.0 \\
\hline N.M. & 63.7 & 55.3 & 62.1 & 53.7 & 64.3 & 59.6 \\
\hline A.L. & 63.5 & 50.3 & 64.4 & 56.6 & 64.1 & 62.0 \\
\hline B.L. & 64.8 & 51.5 & 64.4 & 56.0 & 68.0 & 62.7 \\
\hline G.H. & 63.8 & 49.5 & 61.0 & 52.7 & 63.2 & 54.5 \\
\hline G.K. & 64.2 & 51.2 & 63.6 & 55.8 & 62.0 & 60.5 \\
\hline C.S. & 63.1 & 50.3 & 64.7 & 54.4 & 64.7 & 56.0 \\
\hline D.C. & 62.9 & 50.7 & 53.3 & 49.8 & 61.5 & 57.0 \\
\hline Mean & 63.1 & 51.4 & 62.4 & 53.9 & 63.9 & 58.2 \\
\hline
\end{tabular}

Note-IAA, interaural attenuation.

In prior studies comparing older listeners with clinically significant degrees of presbycusis to younger listeners with normal audiograms, small but significant effects of group on the size of the MLD were found (Jerger, Brown, \& Smith, 1984; Novak \& Anderson, 1982; Olsen, Noffsinger, \& Carhart, 1976; Stubblefield \& Goldstein, 1977; but see Kelly-Ballweber \& Dobie, 1984, whose younger and older subjects were matched for hearing loss). However, the size of the group effect on MLDs observed here and in the experiment of Grose et al. (1994) is somewhat larger than what has been previously reported in other studies of older listeners with relatively good audiometric thresholds (Jerger et al., 1984; Novak \& Anderson, 1982). A possible explanation for this apparent discrepancy is that the subjects who participated in our experiments were actually in the early stages of presbycusis, with pure-tone thresholds that did not meet strict criteria for normal hearing at the high frequencies. The criterion for normal hearing used by Jerger and his colleagues was that pure-tone air-conducted thresholds from .25 to $8 \mathrm{kHz}$ had to be less than or equal to $20 \mathrm{~dB} \mathrm{HL}$, and the criterion employed by Novak and Anderson was that pure-tone air-conducted thresholds from .25 to $3 \mathrm{kHz}$ had to be less than or equal to $20 \mathrm{~dB}$ HL. The criterion in the present study was that pure-tone air-conducted thresholds in the better ear had to be less than or equal to $25 \mathrm{dBHL}$ from .25 to $2 \mathrm{kHz}$, and some subjects had ele- vated thresholds at higher frequencies (Pichora-Fuller \& Schneider, 1991). In addition, it is worth noting that the subjects in the present study were older than those in the study of Jerger and his colleagues, in which the oldest subject was only 69 years old, and that Novak and Anderson had half as many subjects in their older normalhearing group as we did in our experiments. As in our study, the subjects of Grose et al. had hearing loss above $2 \mathrm{kHz}$, and their average age was slightly higher than the average age of our subjects. Our results and those of Grose et al. should be taken as characteristic of older individuals in the very early stages of presbycusis, even though this degree of hearing loss may not be considered to be clinically significant and would certainly not warrant recommendation of a hearing aid.

It also seems unlikely that procedural differences can account for the differences between our studies and previous studies. There is no reason to expect that response biases would account for the larger age effect on MLD in our studies because, unlike most of the other researchers (e.g., Jerger et al., 1984; Olsen et al., 1976; Stubblefield \& Goldstein, 1977), who used a Békésy tracking method, we used the 2I2-AFC procedure, which controls for response biases. Moreover, Novak and Anderson used a 2I2AFC procedure similar to ours, but still did not find a significant effect of age on MLD.

A stimulus factor that might account, in part, for the apparent discrepancy between studies is the temporal relation between the target and the masker. Previous studies (Jerger et al., 1984; Novak \& Anderson, 1982) employed continuously presented maskers. It was demonstrated in our first study (Pichora-Fuller \& Schneider, 1991) that the age effect on MLD is diminished although not eliminated when continuous rather than burst masking noise is used. Consequently, we might expect the age effect observed by other investigators to be smaller than the one observed in the present experiments because other investigators used continuously presented masking noise. However, like us (Pichora-Fuller \& Schneider, 1991), Grose et al. (1994) found a significant age effect on MLD using a continuous masker, so the failure to find significant age effects cannot be due solely to whether the masker is presented continuously or in a burst.

The present data and a review of previous studies suggest that a reduction in the size of the MLD in the over60 age group may be related to the degree of hearing loss in older adults. However, studies of individuals with a significant degree of sensorineural hearing loss indicate that although reduced MLDs tend to be associated with sensorineural hearing loss, they are not an inevitable consequence of such hearing losses. Koehnke, Culotta, Hawley, and Colburn (1995), who studied 11 listeners with sensorineural hearing loss, noted that most, but not all, of these listeners had smaller MLDs at $500 \mathrm{~Hz}$. Gabriel, Koehnke, and Colburn (1992) found normal MLDs in 2 individuals with bilaterally symmetrical, high-frequency noise-induced sensorineural hearing loss. Thus, reduced MLDs are not an inevitable consequence of sensorineural hearing loss. Overall, the results suggests that there need 


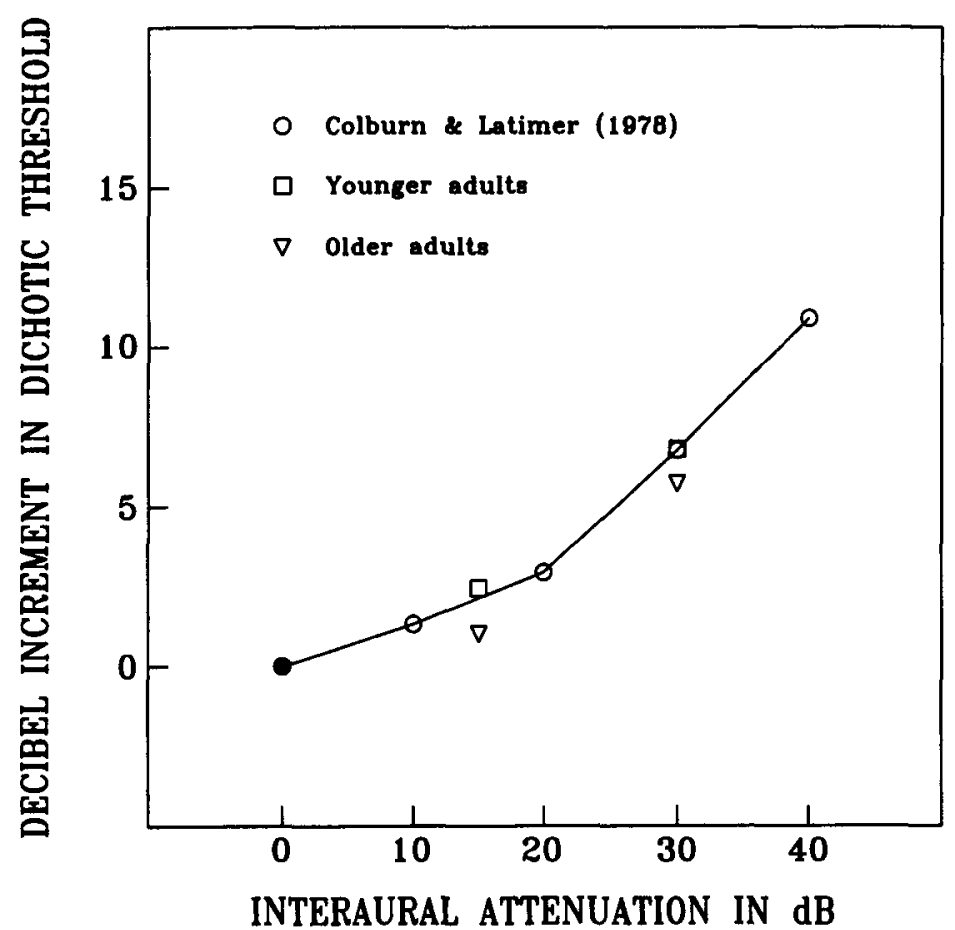

Figure 3. Decibel increase in dichotic threshold as a function of the amount of IAA for the younger and older subjects in the present study, and for the subjects in the study of Colburn and Latimer (1978), who were tested in dichotic conditions nearly identical to ours (see note 3 ). The filled circle represents the reference level for the increase in dichotic threshold.

not be a strong linkage between binaural performance and threshold elevation, leaving open the possibility that deficits in binaural processes may result from underlying abnormalities other than those responsible for threshold elevation.

Schneider (1997), in a recent review of the aging auditory system, presented both physiological and psychophysical evidence suggesting a loss of neural synchrony with age that may occur independently of threshold hearing loss. As Pichora-Fuller and Schneider (1991, 1992) noted, such age-related reductions in neural synchrony could account for the reduced MLDs in older listeners. To see why this is the case, recall that in order for binaural unmasking to occur, the signal plus noise in the right ear must be compared to the signal plus noise in the left ear. If the temporal alignment of the left- and rightear responses is disturbed, as it would be if there was a reduction in neural synchrony, then the ability of the auditory system to unmask the signal would necessarily be reduced. Thus, one of the factors that may be contributing to poorer binaural performance in older adults may be a loss of neural synchrony with age.

\section{REFERENCES}

Blodgett, H, C., JefFress, L. A., \& Whitworth, R. H. (1962). Effect of noise at one ear on the masked threshold for tone at the other. Journal of the Acoustical Society of America, 34, 979-981.

CanAhl, J. A., \& SMaLl, A. M. (1965). Masking-level differences as a function of masker level. Journal of the Acoustical Society of America, 38, 928.

COlburn, H. S., \& Latimer, J. S. (1978). Theory of binaural interaction based on auditory-nerve data: III. Joint dependence on interaural time and amplitude differences in discrimination and detection. Journal of the Acoustical Society of America, 64, 95-106.

DierCKS, K. J., \& JeFFress, L. A. (1962). Interaural phase and the absolute threshold for tone. Journal of the Acoustical Society of America, 34, 981-984.

Dolan, T. R. (1968). Effect of masker spectrum level on masking-level differences at low signal frequencies. Journal of the Acoustical Society of America, 44, 1507-1512.

Dolan, T. R., \& RoBinson D. E. (1967). Explanation of masking-level differences that result from interaural intensive disparities of noise. Journal of the Acoustical Society of America, 42, 977-981.

Durlach, N. I., \& Colburn, H. S. (1978). Binaural phenomena. In E. C. Carterette \& M. P. Friedman (Eds.), Handbook of perception: Hearing (Vol. 4, pp. 365-466). London: Academic Press.

Gabriel, K. J., Koennke, J., \& Colburn, H. S. (1992). Frequency dependence of binaural performance in listeners with impaired binaural hearing. Journal of the Acoustical Society of America, 91, 336-347.

GREEN, D. M. (1966). Interaural phase effects in the masking of signals of different durations. Journal of the Acoustical Society of America, 39, 720-724.

Grose, J. H., Poth, E. A., \& Peters, R. W. (1994). Masking level differences for tones and speech in elderly listeners with relatively normal audiograms. Journal of Speech \& Hearing Research, 37, 422-428.

HaLl, J. W., \& HARVEY, A. D. G. (1984). $\mathrm{N}_{0} \mathrm{~S}_{0}$ and $\mathrm{N}_{0} \mathrm{~S}_{\pi}$ thresholds as a function of masker level for narrow-band and wideband masking noise. Journal of the Acoustical Society of America, 76, 1699-1703.

HALL, J. W., \& HARVEY, A. D. G. (1985). The binaural masking level difference as a function of frequency, masker level and masking bandwidth in normal-hearing and hearing-impaired listeners. Audiology, 24, 25-31. 
Hall, J. W., Tyler, R. S., \& Fernandes, M. A. (1983). Monaural and binaural auditory frequency resolution measured using bandlimited noise and notched-noise masking. Journal of the Acoustical Society of America, 73, 894-898.

HENNING, G. B., \& ZWICKER, E. (1984). Effects of the bandwidth and level of noise and of the duration of the signal on binaural maskinglevel differences. Hearing Research, 14, 175-178.

Hirsh, I. (1948). The influence of interaural phase on interaural summation and inhibition. Journal of the Acoustical Society of America, 20, 536-544.

Jerger, J., Brown, D., \& Smith, S. (1984). Effect of peripheral hearing loss on the masking level difference. Archives of Otolaryngology, 110, 290-296.

Kelly-Ballweber, D., \& Dobie, R. A. (1984). Binaural interaction measured behaviorally and electrophysiologically in younger and older adults. Audiology, 23, 181-194.

Koehnke, J., Culotta, C. P., Hawley, M. L., \& Colburn, H. S. (1995). Effect of reference interaural time and intensity differences on binaural performance in listeners with normal and impaired hearing. Ear \& Hearing, 16, 331-353.

LANGFORD, T. L., \& JeFFRESS, L. A. (1964). Effect of noise crosscorrelation on binaural signal detection. Journal of the Acoustical Society of America, 36, 1455-1458.

MCFADDEN, D. (1968). Masking-level differences determined with and without interaural disparities in masker intensity. Journal of the Acoustical Society of America, 44, 212-223.

NovaK, R. E., \& ANDERSON, C. V. (1982). Differentiation of types of presbycusis using the masking-level difference. Journal of Speech \& Hearing Research, 25, 504-508.

Olsen, W. O., Noffsinger, D., \& Carhart, R. (1976). Masking level differences encountered in clinical populations. Audiology, 15, 287-301.

Pichora-Fuller, M. K., \& SChNeider, B. A. (1991). Masking-level differences in the elderly: A comparison of antiphasic and time-delay dichotic conditions. Journal of Speech \& Hearing Research, 34, 14101422.

Pichora-Fuller, M. K., \& Schneider, B. A. (1992). The effect of interaural delay of the masker on masking-level differences in younger and older adults. Journal of the Acoustical Society of America, 91, 2129-2135.

SCHNEIDER, B. (1997). Psychoacoustics and aging: Implications for everyday listening. Journal of Speech-Language Pathology \& Audiology, 21, 111-124.

Stubblefield, J. H., \& Goldstein, D. P. (1977). A test-retest reliability study on clinical measurement of masking level differences. $A u$ diology, 16, 419-431.

Willot, J. F. (1991). Aging and the auditory system: Anatomy, physiology, and psychophysics. San Diego: Singular.

Wolfram, S. (1991). Mathematica: A system for doing mathematics by computer. Redwood City, CA: Addison-Wesley.

Working Group on SPEech Understanding \& Aging \& Committee on Hearing, Bioacoustics, \& Biomechanics (1988). Speech understanding and aging. Journal of the Acoustical Society of America, 83, 859-895.

Yost, W. A. (1988). The masking-level difference and overall masker level: Restating the internal noise hypothesis. Journal of the Acoustical Society of America, 83, 1517-1521.

\section{NOTES}

1. MLDs are largest when the interaural time delay in the noise is equal to half the period of the tonal signal (Langford \& Jeffress, 1964).

2 . We use subscripts to designate the interaural phase and time differences in the $S_{\pi} N_{\pi}$ and $S_{\pi} N_{\pi \tau}$ conditions, but we have not added a subscript that specifies the degree of interaural attenuation.

3. Colburn and Latimer (1978) intended to determine dichotic thresholds in $\mathrm{S}_{\pi \tau} \mathrm{N}_{\tau}$ (see their Equation 2). However, they inadvertently produced the time delay by a combination of a phase shift ( $\pi$ radians) and a pure time delay. Note that a phase shift of $180^{\circ}$ in both signal and noise produces $\mathrm{S}_{\tau} \mathrm{N}_{\pi \tau}$. Finally, note that when $\tau=1 \mathrm{msec}, \mathrm{S}_{\tau}=1 \mathrm{~N}_{\pi, \tau=1}$ is equivalent to $S_{\pi} N_{\pi, \tau=1}$ when $S$ is a $500-\mathrm{Hz}$ pure tone, which is exactly the condition that we used. The closest value of $\tau$ to $1 \mathrm{msec}$ that they used was $0.8 \mathrm{msec}$. A pure time delay of $-0.8 \mathrm{msec}$ and a phase shift of $180^{\circ}$ corresponds to the condition that they showed as $0.2 \mathrm{msec}$ in their Table 1 . Therefore, the subset of data collected by Colburn and Latimer that comes closest to our condition is the $\pm 0.2-\mathrm{msec}$ condition in their Table 1. We averaged these data over subjects to produce the data shown in Figure 3.

(Manuscript received February 21, 1996; revision accepted for publication October 17, 1997.) 